\title{
Nanograting Orientation Influence on Stress Induced by Femtosecond Laser in Fused Silica
}

\author{
Audrey Champion ${ }^{1}$, Martynas Beresna ${ }^{2}$, Peter G. Kazansky ${ }^{2}$, Yves Bellouard $^{1}$ \\ ${ }^{1}$ Department of Mechanical Engineering Eindhoven University of Technology \\ Den Dolech 2, 5612 AZ Eindhoven, The Netherlands. \\ ${ }^{2}$ Optoelectronics Research Centre, University of Southampton, SO17 1BJ, United Kingdom. \\ A.Champion@tue.nl
}

\begin{abstract}
Under certain conditions, femtosecond lasers can be used to introduce selforganized nanogratings in the bulk of fused silica. Here, we report that the nanogratings orientation influences the induced stress distribution around laser affected zones.
\end{abstract}

\section{Introduction}

Femtosecond laser exposure in fused silica leads to various structural modifications [1]. These modifications introduce stress within the material [2]. Among the structural changes, of particular interest are the selforganized nanogratings [3], that are found under certain conditions of exposure [4]. These nanogratings form perpendicular to the electric field of the laser light [3]. Recently, we showed that the formation of nanogratings is also associated with a localized volume expansion [5], consistent with the hypothesis of porous material forming inside the laser affected volume [6].

In this paper, we demonstrate that the stress induced by the nanogratings volume expansion also depends on their orientation, itself controlled by the laser polarization. This result is important for the machining of silica substrates in general, since the stress induced by the laser affects not only the etching of the material [7], but also the optical quality of photonics devices produced with this method [8].

\section{Methodology}

Retardance is created by permanent induced stress after laser irradiation, in and around the laser affected zone. From the measured retardance, the equivalent difference principal stress is expressed by:

$$
\sigma_{\theta}-\sigma_{r}=\frac{R}{T\left(C_{2}-C_{1}\right)}
$$

where (C2-C1) are the elasto-optic coefficients equal to $3.55 \times 10^{-12} \mathrm{~Pa}^{-1}$ for fused silica, $\mathrm{T}$ the sample thickness and $\mathrm{R}$ the retardance.

Retardance measurement in the immediate vicinity of the nanogratings is difficult to measure due to the sharp retardance intensity decay over a few microns. To overcome this limitation, we enlarge the span of the stressed region using a dedicated geometry pattern called 'sun pattern'. In Fig. 1 left, the entire top view of the sun pattern and a transverse portion is shown to create the desired profile. Fig. 1 right illustrates the measured retardance distribution around the sun pattern when the linear polarization of the laser keeps the same direction while the writing direction is varied with $15^{\circ}$ angle increment. If the laser polarization is kept constant while writing the sun-pattern, the nanogratings will gradually turn with the line directions, as illustrated in Fig. 1 left (red lines in the top view).
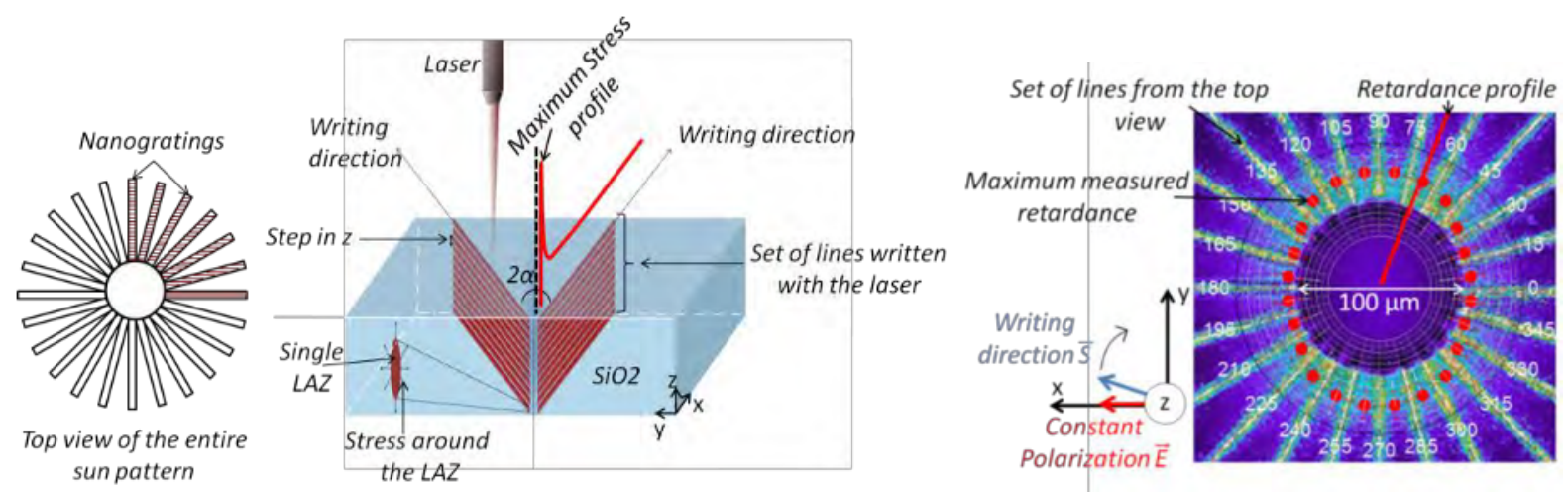

Fig. 1 Left: Top view and transverse portion of the sun pattern. Nanogratings have a constant orientation compare to the writing direction. Right: Maximum retardance measurement around the entire sun pattern for angles $15^{\circ}$ increment. 
We measure the maximum retardance around the entire sun-pattern (red dots in Fig. 1 right). We observe a non-homogenous stress distribution around the circular pattern, meaning that the induced stress depends on the angle.

To capture this orientation dependence and to estimate the stress created at the interface of laser affected zones, we first simulate the influence of the nanogratings orientation on a single line assuming that the nanogratings expands locally. This is used for estimating the average stress $\sigma_{\theta}$ along a single line with nanogratings of a given orientation. This average stress is then used as input for the complete model that considers the full sun-pattern.

Results are shown in Fig. 2. The nanogratings shape and periodicity used in the simulation are defined from scanning electron microscopy observations. As boundary conditions, we assume that a homogeneous pressure that we call $\sigma_{\mathrm{NG}}$ is applied all over a segment of the nanogratings (black arrows in Fig. 2 right). By calibrating the finite element model with the experimental observations reported in Fig. 1, we found that according this model, the stress around single nanogratings cells can be as high as $2 \mathrm{GPa}$. The complete simulation is performed with an increment angle of $15^{\circ}$ between $0^{\circ}$ and $90^{\circ}$. The average stress is simulated for every angle.
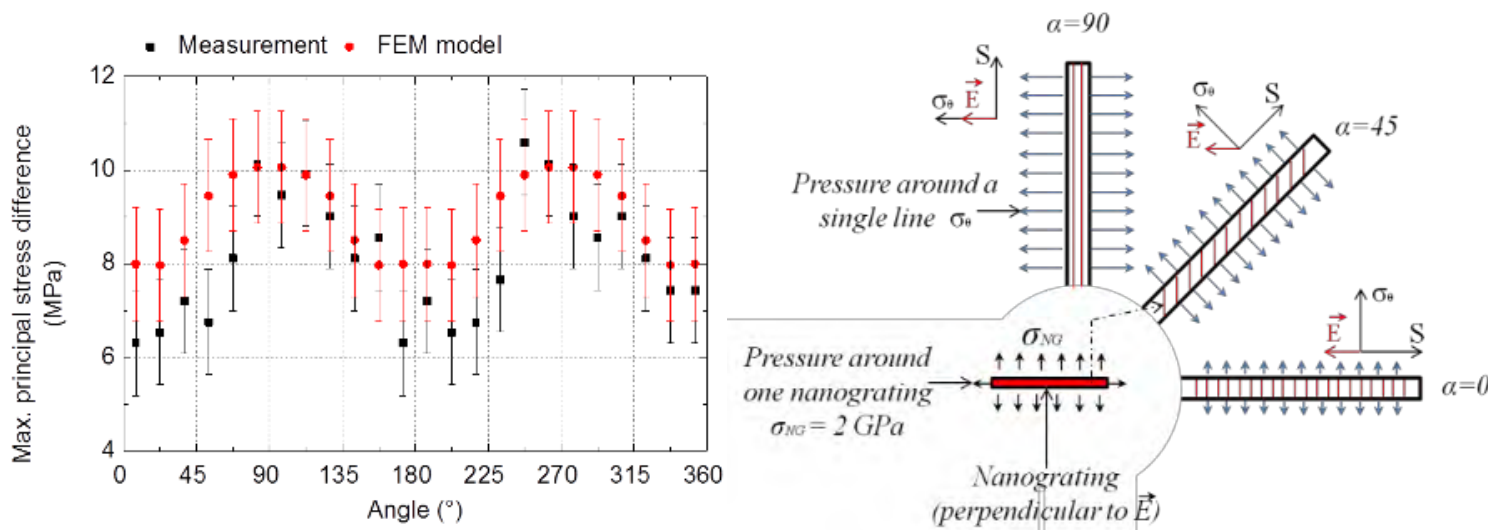

Fig. 2 Left: Maximum simulated and measured stress according to the angle with $15^{\circ}$ increments. Right: Nanograting orientation influence around a single line for $\alpha=0^{\circ}, 45^{\circ}$ and $90^{\circ}$.

As shown in Fig. 2 left, there is a good agreement between the model and the experiments which further validates the hypothesis of volume expansion. It also shows that the nanogratings orientation and therefore the laser polarization, has a non-negligible influence on the stress induced in the material.

\section{Conclusion}

We demonstrate that the nanograting orientation is important in term of retardance and stress distribution around the laser affected zone. The stress distribution can be directly measured with the retardance and controlled by the polarization of the laser.

This result has practical importance for the manufacturing with femtosecond laser in the regime where nanogratings are found, since etching profile is affected by stress distribution as well as optical properties.

\section{References}

[1] K. M. Davis, K. Miura, N. Sugimoto, and K. Hirao, "Writing waveguides in glass with a femtosecond laser," Opt. Lett. 21, 1729-1731 (1996).

[2] Y. Bellouard, T. Colomb, C. Depeursinge, M. Dugan, A. A. Said, and P. Bado, "Nanoindentation and birefringence measurements on fused silica specimen exposed to low energy femtosecond pulses, " Opt. Express 14, 8360 (2006).

[3] Y. Shimotsuma, P. G. Kazansky, J. R. Qiu, and K. Hirao, "Self-organized nanogratings in glass irradiated by ultrashort light pulses," Phys Rev. Lett. 91, 247705 (2003).

[4] C. Hnatovsky, R. S. Taylor, P. P. Rajeev, E. Simova, V. R. Bhardwaj, D. M. Rayner, and P. B. Corkum, "Pulse duration dependence of femtosecond-laser-fabricated nanogratings in fused silica," Applied Physics Letters 87, 014104-014104-3 (2005).

[5] A. Champion and Y. Bellouard, "Direct volume variation measurements in fused silica specimens exposed to femtosecond laser," Optical Materials Express 2, 789 (2012).

[6] B. Poumellec, M. Lancry, J.C. Poulin, and S. Ani-Joseph, "Non reciprocal writing and chirality in femtosecond laser irradiated silica," Opt. Express 16, 18354-18361 (2008).

[7] S. Rajesh and Y. Bellouard, "Towards fast femtosecond laser micromachining of fused silica: The effect of deposited energy," Opt. Express 18, 21490-21497 (2010).

[8] M. Beresna, M. Gecevičius, P.G. Kazansky, and T.Gertus' "Radially polarized optical vortex converter created by femtosecond laser nanostructuring of glass," Appl. Phys. Lett. 98, 201101 (2011). 